INL/EXT-18-44749 Rev. 0

February 2018

\title{
Improved LWR Fuel Rod Mechanics Models
}

\section{M3MS-18IN0201015}

NEAMS Milestone report

B. W. Spencer

R. J. Gardner

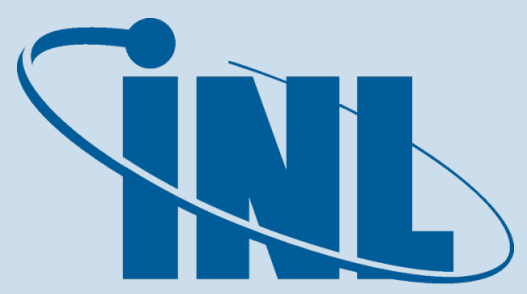

Idaho National Laboratory 


\section{NOTICE}

This information was prepared as an account of work sponsored by an agency of the U.S. Government. Neither the U.S. Government nor any agency thereof, nor any of their employees, makes any warranty, express or implied, or assumes any legal liability or responsibility for any third party's use, or the results of such use, of any information, apparatus, product, or process disclosed herein, or represents that its use by such third party would not infringe privately owned rights. 
INL/EXT-18-44749 Rev. 0

\section{M3MS-18IN0201015 \\ Improved LWR Fuel Rod Mechanics Models}

NEAMS Milestone report

B. W. Spencer

R. J. Gardner

February 2018

Idaho National Laboratory

Fuel Modeling and Simulation Department Idaho Falls, ID 83415

Prepared for the

U.S. Department of Energy

Office of Nuclear Energy

Under U.S. Department of Energy-Idaho Operations Office

Contract DE-AC07-99ID13727 


\begin{abstract}
There has been an ongoing effort to improve the mechanical constitutive models used in the BISON fuel performance code to improve their robustness and accuracy. This report documents recent work that was directed at improving the smeared cracking model used to represent fracture, especially for application to axisymmetric two-dimensional representations of light water reactor (LWR) fuel. It also demonstrates the application of this model in axisymmetric fuel rod simulations, including an example problem and a full-length axisymmetric LWR fuel rod model with available profilometry data in the BISON assessment test suite.

The work described in this report was performed under funding from the Nuclear Energy Advanced Modeling and Simulation (NEAMS) program. This report is issued in satisfaction of the Level 3 milestone M3MS-18IN0201015 on improving light water reactor (LWR) fuel rod mechanics models in the INL Engineering-Scale Fuel Performance (BISON) Work Package.
\end{abstract}




\section{Contents}

\begin{tabular}{ll}
\hline Introduction & 1
\end{tabular}

1 Smeared Cracking Model Improvements 2

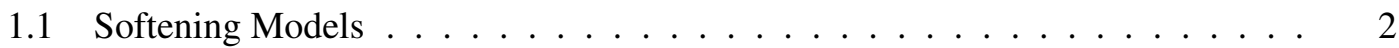

1.2 $\quad$ Investigation of Softening Models for 2D Axisymmetric Representations . . . . 4

1.3 Enhancements to the Smeared Cracking Model . . . . . . . . . . . . . . 7

2 Application of Smeared Cracking to BISON Example and Assessment Problems 9

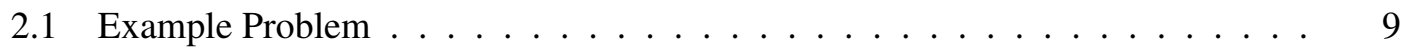

2.1 .1 Simulation Description . . . . . . . . . . . . . . . . . . 9

2.1 .2 Results . . . . . . . . . . . . . . . . . . . . . 9

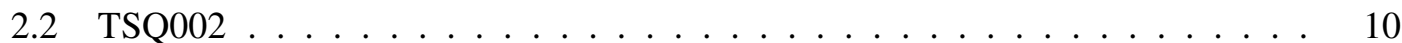

2.2 .1 Simulation Description . . . . . . . . . . . . . . . . . . . 10

2.2 .2 Results . . . . . . . . . . . . . . . . . . . . 11

$\begin{array}{lll}3 & \text { Summary } & 16\end{array}$

\begin{tabular}{ll}
\hline Acknowledgments & 17
\end{tabular}

\begin{tabular}{ll}
\hline Bibliography & 18
\end{tabular} 


\section{Introduction}

In the light water reactor (LWR) environment, the mechanical behavior of oxide nuclear fuel is affected by a number of effects, including creep, swelling, thermal expansion, and cracking. Including the effects of cracking in the nuclear fuel is important because it effects the overall radial displacement of the fuel pellets, and has a major effect on the stress state within the pellet. If cracking is not accounted for, the stress state in the fuel will be very un-realistic, severely affecting the accuracy of models for other nonlinear constitutive behavior such as creep and hot pressing.

In engineering simulations of nuclear fuel, it is common to use a smeared cracking approach, in which cracking is represented by a softening stress-strain law at material integration points. The objective of this technique is not to accurately resolve individual discrete cracks, but to represent the effects of cracking on quantities of engineering interest such as average stress fields and structural deformation.

BISON's smeared cracking model is based on the fixed smeared cracking model originally proposed by Rashid [1]. Work in previous years under the NEAMS program has improved the robustness of BISON's implementation of the smeared cracking model, and migrated that model to the TensorMechanics model, where all current mechanical model development in BISON is taking place [2].

This report documents recent work that was directed at improving the smeared cracking model used to represent fracture, especially for application to axisymmetric two-dimensional representations of light water reactor (LWR) fuel. It also demonstrates the application of this model in axisymmetric fuel rod simulations, including an example problem and a full-length axisymmetric LWR fuel rod model with available profilometry data in the BISON assessment test suite. 


\section{Smeared Cracking Model Improvements}

The smeared cracking approach represents the effects of cracking through a strain-softening constitutive model run at the standard finite element integration points. When one of the principal stresses exceeds a tensile failure limit, the model converts to an orthotropic model that has a softening response in the direction of cracking. The orientation of the first crack is fixed, and up to two additional cracks are allowed to form at a given material point in directions orthogonal to the pre-existing cracks.

The softening response is obtained in the material model by making two key modifications to the material response. First, the elasticity tensor in the coordinate system of the crack is modified so that the stiffness is reduced in the direction of cracks. This damaged elasticity tensor, based on the maximum strain in the previous time step, is used to compute an updated stress during each material evaluation. The second modification made to the response is that the predicted stresses in the direction of cracking are adjusted to follow a defined softening function.

\subsection{Softening Models}

The choice of the softening function has an important impact on the response of the cracked material. A major part of the recent development efforts on this model involved improving the flexibility of the selection of softening models. A short discussion of the softening models is provided here.

BISON provides three options for representing strain-softening behavior after crack initiation, shown graphically in Figure 1.1. The exponential softening model employs an exponential function to describe the softening behavior:

$$
\sigma=f_{t}\left[r_{t}+\left(1-r_{t}\right) \exp \left(\frac{\alpha\left(\varepsilon-\varepsilon_{c r}\right)}{f_{t}}\right)\right]
$$

where $f_{t}$ is the tensile strength of the material, $r_{t}$ is a residual tensile stress that remains after the material has fully softened, $\alpha$ is the initial slope of the softening curve upon fracture initiation, $\varepsilon$ is the strain in the cracking direction, and $\varepsilon_{c r}$ is the strain in the cracking direction at crack initiation. The exponential softening model represents the softening behavior of quasi-brittle materials such as concrete, and is also widely used for nuclear fuel.

The power law softening model is based on the idea that the material represented by an integration points can be cracked multiple times in a given direction, and each time a new crack forms, 
Figure 1.1: Stress-strain behavior of the softening models provided by BISON

the stiffness is reduced by a factor. The initial stiffness of the material is the Young's modulus, $E$. If the stiffness reduction factor is $c$ and the number of cracks is $n$, this has the following form:

$$
\sigma=E(c)^{n}
$$

The post-peak response with this model is represented by the saw-tooth behavior shown in Figure 1.1. where the material has linear behavior until a new crack forms, after which it abruptly loses a significant portion of its strength. The primary motivation of this model is representing the hoop response in axisymmetric models, where a number of radial cracks form through the ring of material represented by a single material integration point.

Finally, the abrupt softening model simply drops the stress to zero, or a residual value close to zero, after the tensile strength is exceeded. This model is intended to represent a brittle response.

There are two important aspects of the smeared softening model that must be considered:

- Fracture energy is a material property that should be correctly represented by the chosen softening model. The fracture energy released in an element is equal to the total area under the hardening and softening portions of the stress/strain response times a finite element characteristic length in the direction of cracking. This should be accounted for in the selection of the softening curve, and ideally, the characteristic length should be computed automatically so that the softening curve is specified using the fracture energy as an input parameter.

- In two-dimensional models of fracturing material, different softening models are appropriate for the out-of-plane response than the in-plane response. For 3D models and for the 
in-plane response in 2D models, the exponential or abrupt softening models are appropriate, while for the out-of-plane response, the power law model is likely more appropriate in axisymmetric models.

\subsection{Investigation of Softening Models for 2D Axisymmetric Representations}

The power law model has been available for some time in BISON, but a study to determine whether it is appropriate had not yet been performed. To determine an appropriate softening model for use in the out-of-plane (hoop) direction in axisymmetric models, the following procedure is proposed:

1. Develop a planar cross-section model of nuclear fuel that includes an accurate representation of in-plane cracking.

2. Subject that model to conditions typical of nuclear fuel under a ramp up to full power, which will induce cracking.

3. Extract hoop stress and strain averaged over a set of concentric rings with increasing radius to capture the response of the fuel as a function of radius.

4. Examine these results to use as a basis for a softening model for the hoop direction in axisymmetric models.

To this end, a 2D planar cross-section model of representative LWR fuel was developed, as shown in Figure 1.2. The extended finite element method (XFEM) was used to represent discrete cracks in the fuel, using the procedures described in [3]4]. In this model, radial cracks are allowed to form on the outside surface of the fuel and propagate inward. A tensile stress criterion is used for crack initiation and growth, and there is abrupt stress release as cracks propagate into new elements. It would be better to use a fracture energy criterion for crack growth, but this simplified approach was used for an initial proof of this concept.

A set of code objects were developed to compute averages of various quantities of interest over a set of rings going from the inside to the outside of the fuel pellet. In this case, quantities were averaged over 10 concentric rings, going from the centerline to the outer radius of the fuel. Averaging the hoop stress is fairly straightforward - the component of the stress tensor in the hoop direction is simply averaged over a given ring. Computing a meaningful strain quantity is more complicated because cracking is represented by a displacement discontinuity, so the mechanical strain tensor does not account for the effects of cracking. Instead, the hoop strain is computed by averaging the radial component of the displacement divided by the radius for all material points in a given ring. A stress-strain curve obtained for the outermost five rings is shown in Figure 1.4

The objective of this exercise is to understand what an appropriate softening response for an engineering model of the response in the hoop direction should be. Because the strain shown in 


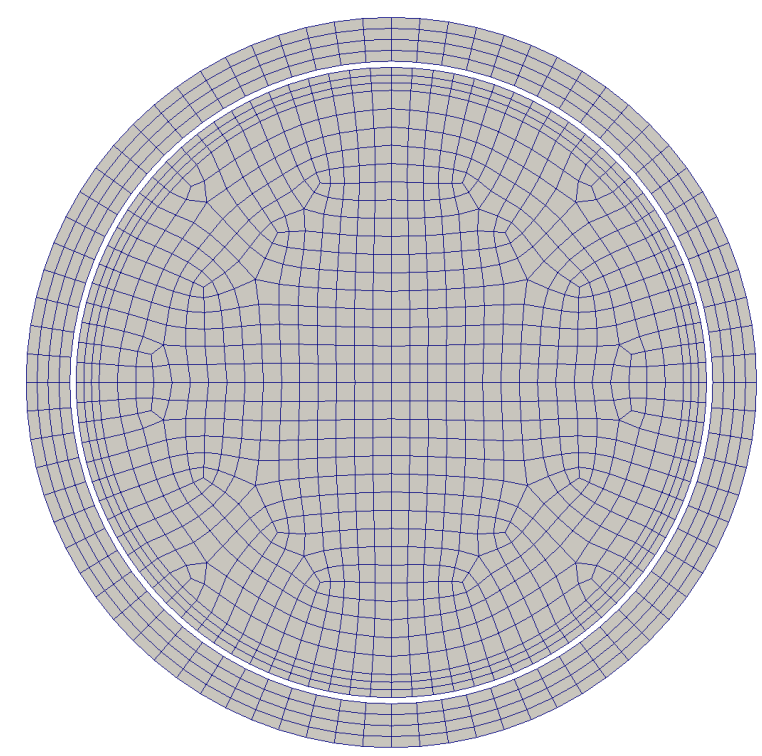

Figure 1.2: Mesh used for 2D planar cross-section model with XFEM

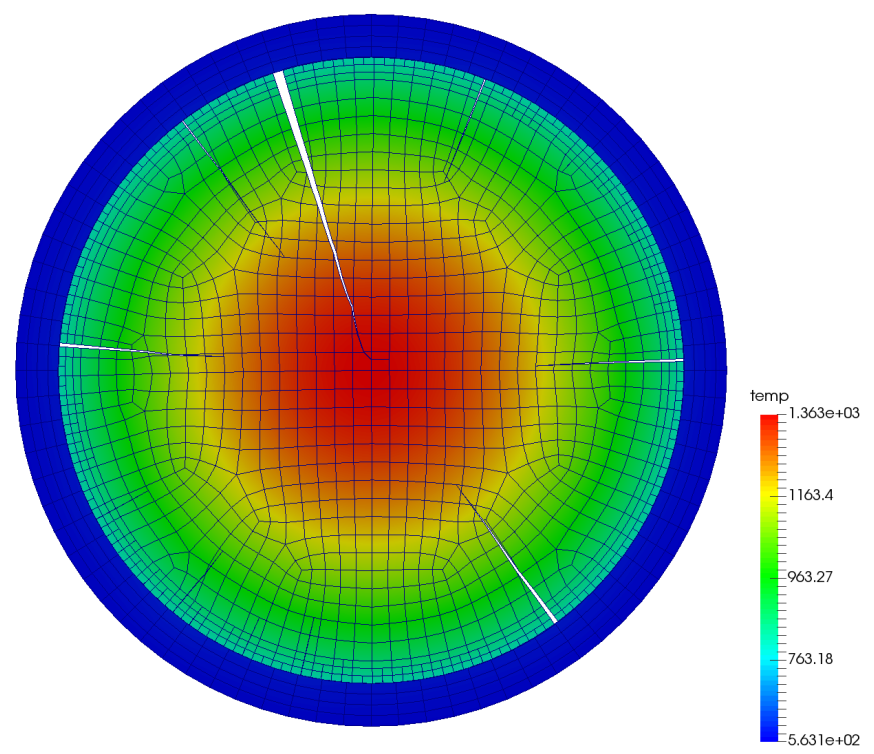

Figure 1.3: Deformed mesh with cracks represented using XFEM at full power $(25 \mathrm{~kW} / \mathrm{M})$ showing temperature contours. Displacements are magnified $2 \mathrm{x}$ to highlight cracks (which also exaggerates the fuel/cladding gap closure) 
Figure 1.4: Averaged hoop stress vs. hoop strain curves obtained for the outermost rings in the planar cross-section model of cracking fuel. Hoop strain is computed as the average radial displacement divided by radius.

Figure 1.5: Averaged hoop stress vs. hoop strain curves obtained for the outermost rings in the planar cross-section model of cracking fuel. Hoop strain is computed as the average radial displacement divided by radius as in Figure 1.4, but the hoop component of the volumetric strain induced by thermal expansion is subtracted from the strain. 
Figure 1.4 includes the effect of thermal expansion, it is over-stated, and the effects of thermal expansion should be removed. This was done by subtracting the average of the hoop component of the strain induced by thermal expansion, and the resulting stress-strain curves are shown in Figure 1.5 . There are clearly flaws in this approach, as the subtracted thermal expansion strains appear to be too large, as evident in the initial negative response. This is likely due to the effects of the out-of-plane response, and will be more thoroughly investigated.

Although there are still some issues to resolve in these plots, they do show that the material exhibits the type of sawtooth post-peak behavior that is represented in the power law softening model. Once the first set of cracks form, the stress abruptly drops to a fraction of its initial value, and the stiffness drops by a consistent amount. Following that, the stress increases nearly linearly with increasing strain, until the next set of cracks forms. This behavior is most evident in the outermost ring, but it is also visible in inner rings. The innermost rings (not shown) are initially under compression, and they exhibit a much more complex behavior. It is important to note that cracks tend to quickly propagate from the outside to the inside, so the inner rings have lower apparent tensile strength than the outer rings. It is also important to realize that although the smeared cracking model considers material points independently, the responses of these rings are not independent of each other, so representing it at local material points will always have some degree of inaccuracy.

\subsection{Enhancements to the Smeared Cracking Model}

Based on the study shown in the previous section, it is clear that separate softening models should be used for the out-of-plane response in 2D models. To that end, the smeared cracking code was re-organized and new features were added to permit the use of different softening models in different directions. A summary of the code changes is provided below:

- A new option (prescribed_crack_directions) was added to the smeared cracking model to allow the user to optionally prescribe the directions of the first, second, and third crack in directions aligned with the Cartesian axes in the original model configuration. In a 2D model, this permits the user to prescribe that the first crack is in the z-direction (out of plane).

- Separate softening models can optionally be prescribed in the 3 cracking directions. The order in which they are prescribed associates them with the first, second, and third cracks. If the first crack is prescribed in the z-direction in a 2D model, for instance, this allows a specific softening model to be used in that direction, and another model to be used in the in-plane directions. This is useful for selecting the power law softening model in axisymmetric simulations. It is also useful for planar or axisymmetric XFEM simulations, where XFEM is used to represent discrete cracking in-plane, but a smeared approach is still used out-of-plane.

- Previously, the crack damage was available for output through the crack_flags material property, which was a size 3 vector whose components initially had a value of 1 , and 
dropped to 0 as the material in the crack directions became fully damaged. The ordering of those components was confusing, as the 3rd component corresponded to the 1st crack. A new property, crack_damage has been added, which indicates the damage (going from 0 to 1 ) in the 3 crack directions in ascending order, which is more user-friendly.

- A bug was found and corrected, in which the crack coordinate system was not being correctly rotated to the current material orientation in finite deformation analyses.

- A number of general cleanup tasks and optimizations of the code were performed to make the code better documented and more readable. 


\title{
2 Application of Smeared Cracking to BISON Example and Assessment Problems
}

\begin{abstract}
Because of historic issues with the smeared cracking model in BISON, most current BISON simulations of LWR fuel represent the fuel as elastic. Now that the smeared cracking model is more robust, the example and assessment problems in the BISON test suite should be updated to include the effects of nonlinear material response, including cracking, creep, and hot pressing. To that end, two problems in the BISON test suite have been updated to include smeared cracking. The first of these is an example problem that models a 10 pellet high smeared fuel stack with common geometries for pellet height and diameter. The second simulation is of a full length fuel rod that is currently in the BISON assessment suite. Previously both of these models represented the fuel using elastic mechanical models.
\end{abstract}

\subsection{Example Problem}

\subsubsection{Simulation Description}

BISON's example problem suite includes axisymmetric models of a smeared fuel stack of 10 pellets and the cladding. The mesh for this problem is shown in Figure 2.1.

To keep this demonstration simple and to focus on the cracking itself, the example problem only models a relatively rapid rise to full power. The initial temperature of the system is $293 \mathrm{~K}$, with no power, and the initial plenum pressure is $2.0 \mathrm{MPa}$. The power is ramped to $25 \mathrm{~kW} / \mathrm{m}$ over $1.0 \mathrm{e} 4 \mathrm{sec}$ at a constant coolant pressure of $15.5 \mathrm{MPa}$. For this demonstration, simulations with and without smeared cracking were performed.

\subsubsection{Results}

The simulation of the ramp to full power completed in 224 seconds on a late 2013 Mac Pro using 4 processors and BISON version e035682f7. For comparison, the elastic fuel version completed in 200 seconds with everything else held equal. Because this demonstration problem does not experience fuel/cladding contact, there are a limited number of quantities of interest to plot. Figure 2.2 shows the end of life (EOL) cracking state of the smeared fuel in the radial and axial directions, respectively, with zero indicating no cracking and one indicating fully cracked material. 


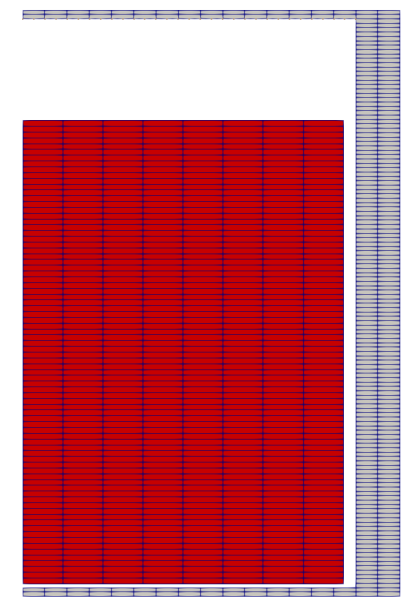

Figure 2.1: Mesh for the 2D axisymmetric 10-pellet example problem. The mesh is scaled 20x in the $\mathrm{x}$-axis.
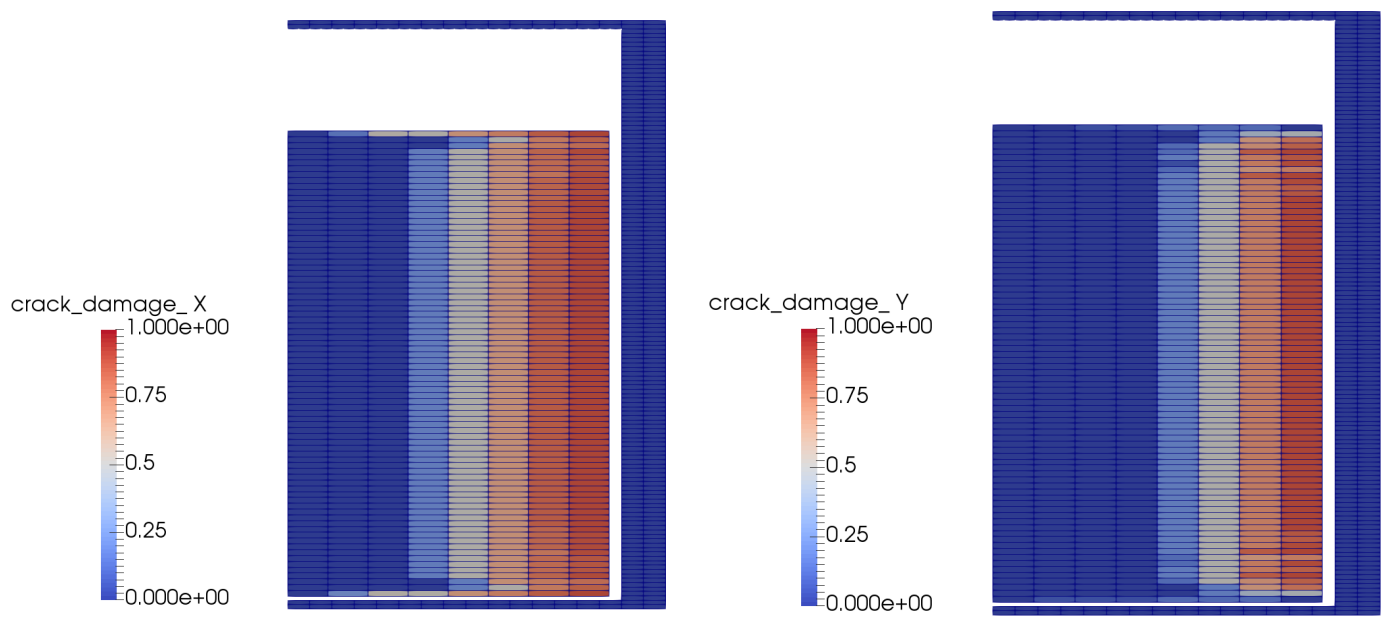

Figure 2.2: Contour of the cracking damage in the radial (left) and axial (right) directions after the ramp to full power in the 10-pellet smeared example problem. The mesh is scaled $20 \mathrm{x}$ in the $\mathrm{x}$-axis.

\subsection{TSQ002}

\subsubsection{Simulation Description}

The TSQ002 experiment was one of a series of experiments that were conducted in the 1980s with the purpose of demonstrating increased discharge burnup though more efficient fuel management. The BISON model of this experiment represents the full length full rod with an active fuel column height of $3.81 \mathrm{~m}$. This rod was irradiated to a burnup of $58 \mathrm{MWd} / \mathrm{kgU}$. [5] 
For the simulation a smeared fuel column was used with geometries provided from the experiment report. As this is a full length fuel rod, significant scaling is required to visualize the whole rod in one figure. Figure 2.3 shows two different views of the same rod so that the full rod and an un-scaled local view of the finite element discretization can be seen.
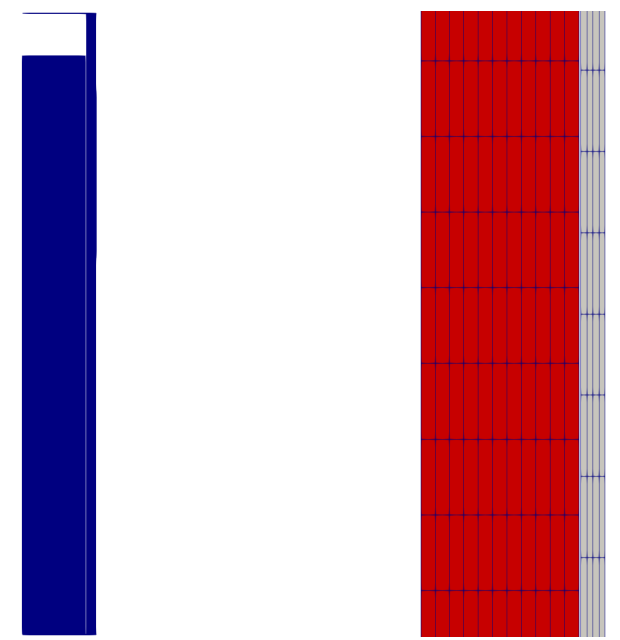

Figure 2.3: Mesh of the TSQ002 fuel rod. The full mesh is shown (left) scaled 100x in the $\mathrm{x}$-axis, and an unscaled section of the mesh is shown (right) at a location near the middle of the rod.

\subsubsection{Results}

All of these simulations were run on Idaho National Laboratory's (INL) Falcon supercomputer. As with the example problem, both an elastic fuel simulation and a smeared cracking simulation were run using 24 processors and BISON version da21d3d. The smeared cracking simulation includes fuel creep, while the elastic simulation does not. The smeared cracking simulation took 27900 seconds ( 7.75 hours) and the elastic fuel simulation took 17600 seconds (4.8 hours). These models also experience contact, which the example problem did not. Contour plots at three different times during the simulation can be seen below in Figures 2.4, 2.5, 2.6, 2.7, 2.8 and 2.9. Note that the green vertical line in the line plot denotes the time in the simulation.

This particular experiment underwent rod profilometry during the post irradiation examination (PIE). Cracking was expected to have a significant effect on the predicted cladding displacement, which is one of the reasons that this rod was selected for the demonstration. The results from the simulations and the EOL PIE can be seen in Figure 2.10 .

As can be seen in Figure 2.10, the BISON elastic fuel model over-predicts the EOL cladding diameter. Due to the addition of radial strains introduced from the smeared cracking model this over prediction is increased slightly, about 4 microns. This result is not unexpected. There are further enhancements currently underway for the UO2 relocation model which will allow for 

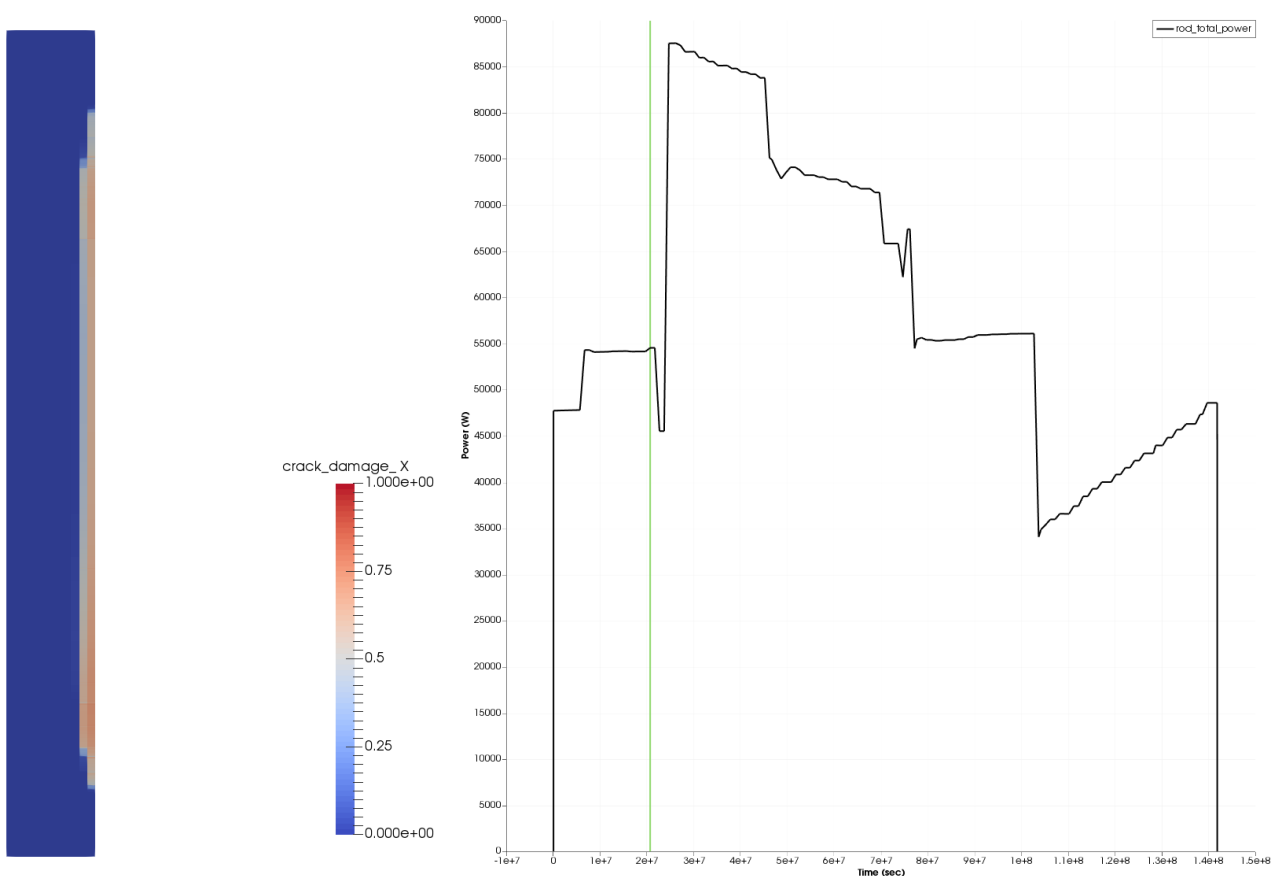

Figure 2.4: Contour plot of the radial cracking (left) and total power history (right) near the beginning of the irradiation. The mesh is scaled 100x in the $\mathrm{x}$-axis.
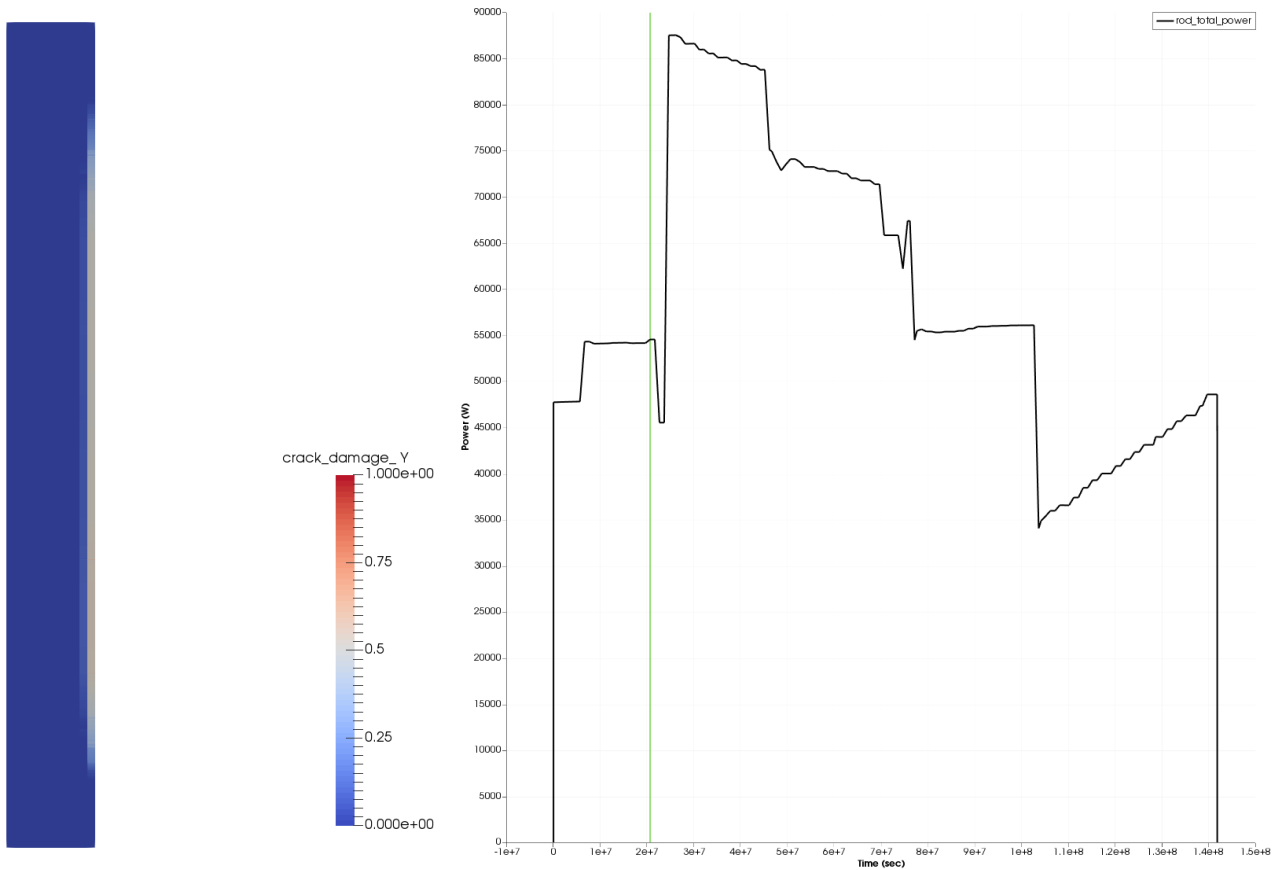

Figure 2.5: Contour plot of the axial cracking (left) and total power history (right) near the beginning of the irradiation. The mesh is scaled 100x in the $\mathrm{x}$-axis. 

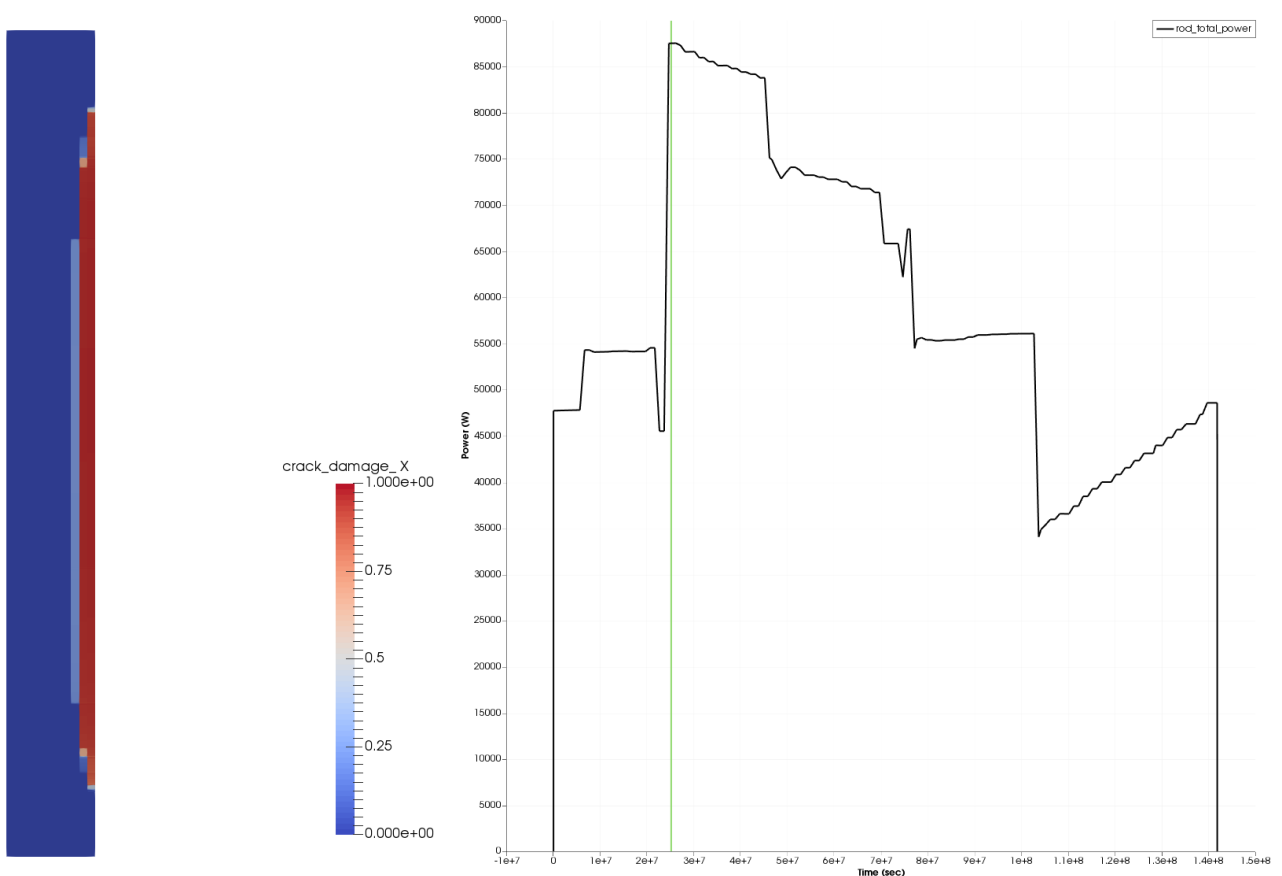

Figure 2.6: Contour plot of the radial cracking (left) and total power history (right) after the second power rise. The mesh is scaled 100x in the $\mathrm{x}$-axis.
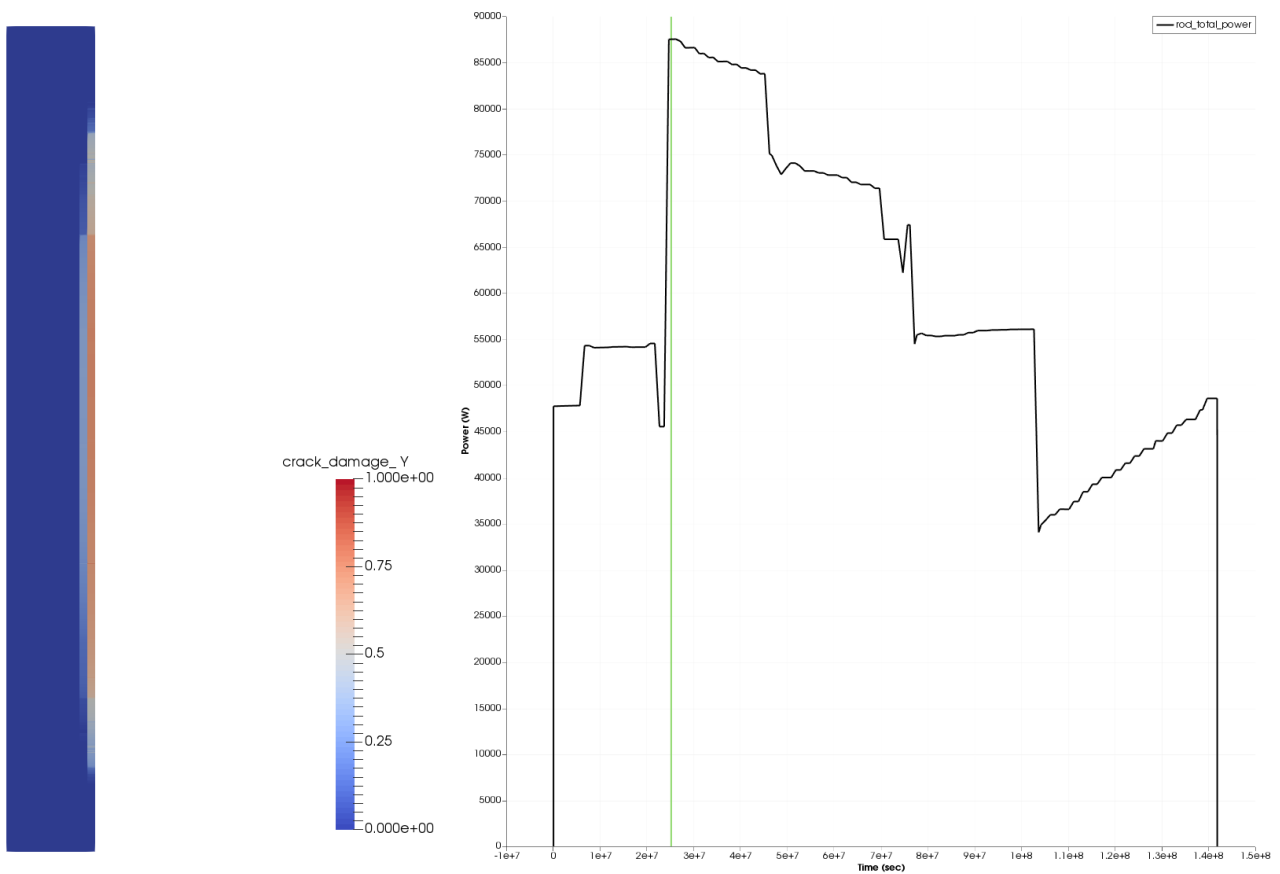

Figure 2.7: Contour plot of the axial cracking (left) and total power history (right) after the second power rise. The mesh is scaled 100x in the $\mathrm{x}$-axis. 

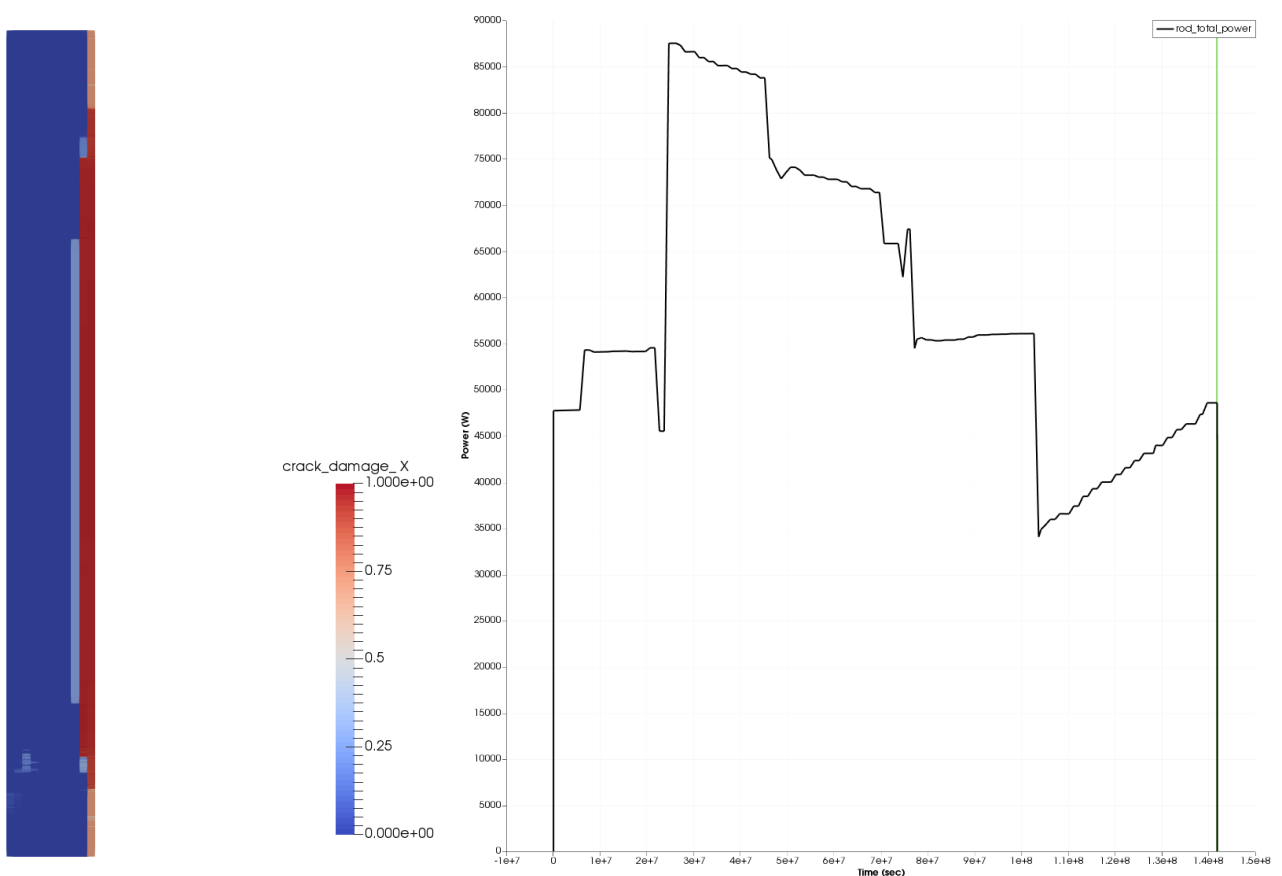

Figure 2.8: Contour plot of the radial cracking (left) and total power history (right) at end of life. The mesh is scaled 100x in the $\mathrm{x}$-axis.
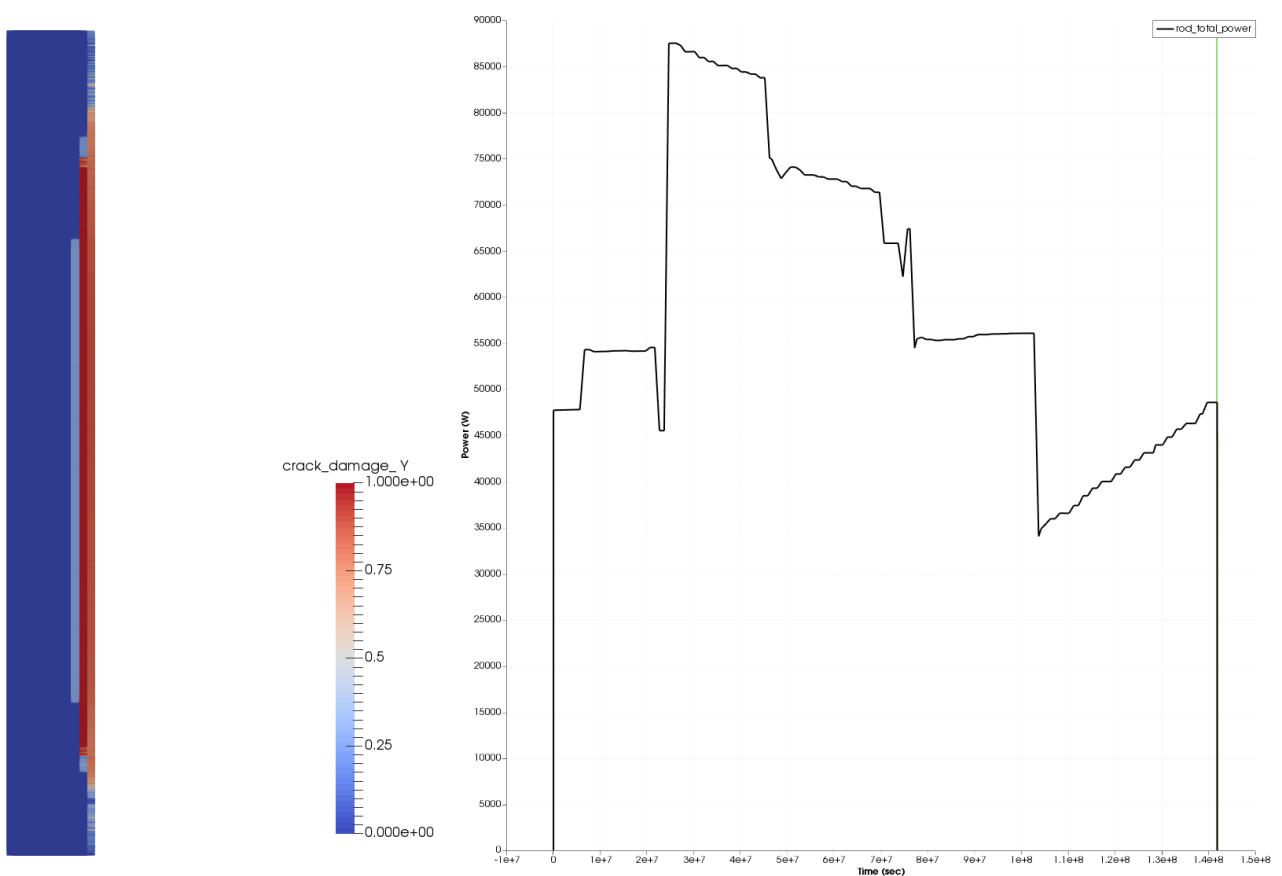

Figure 2.9: Contour plot of the axial cracking (left) and total power history (right) at end of life. The mesh is scaled $100 \mathrm{x}$ in the $\mathrm{x}$-axis. 
Figure 2.10: Comparison of cladding displacement at EOL from the smeared cracking simulation, elastic fuel simulation and the experiment PIE.

relocation recovery. Relocation recovery will account for the fuel strain that is recovered when the fuel and cladding come into contact, which is expected to improve the overall results. 


\section{Summary}

This report documents ongoing work directed at improving the smeared cracking model used to represent fracture, especially for application to axisymmetric two-dimensional representations of light water reactor (LWR) fuel. This includes the following developments:

- A study was performed on a planar 2D cross-section of LWR fuel to understand the softening behavior that should be included in the out-of-plane direction in a 2D axisymmetric model.

- A number of general improvements were made to the smeared cracking model.

- Options were added to the smeared cracking model to permit the selection of different softening models in specified directions.

- Two axisymmetric fuel rod simulations in the BISON test suite were modified to include smeared cracking, and the robustness of that model was demonstrated on those models.

With these recent developments, the smeared cracking capability in BISON is becoming mature and ready for more widespread use. The available models capture important effects, but with further development, the concept of using a $2 \mathrm{D}$ planar cross-section or 3D model to obtain hoop softening behavior could be further developed to improve the softening models used in 2D axisymmetric models. 


\section{Acknowledgments}

This work was funded by the DOE Nuclear Energy Advanced Modeling and Simulation (NEAMS) program. The submitted manuscript has been authored by a contractor of the U.S. Government under Contract DE-AC07-05ID14517. Accordingly, the U.S. Government retains a nonexclusive, royalty free license to publish or reproduce the published form of this contribution, or allow others to do so, for U.S. Government purposes. 


\section{Bibliography}

[1] Y. R. Rashid. Mathematical modeling and analysis of fuel rods. Nuclear Engineering and Design, 29:22-32, 1974.

[2] Benjamin Spencer, Albert Casagranda, Stephanie A. Pitts, and Wen Jiang. Development of 3D oxide fuel mechanics models. Technical Report INL/EXT-17-42856, Idaho National Laboratory, Idaho Falls, ID, July 2017.

[3] Benjamin Spencer, Hai Huang, John Dolbow, and Jason Hales. Discrete modeling of earlylife thermal fracture in ceramic nuclear fuel. In Proceedings of WRFPM 2014, Paper No. 100061, Sendai, Japan, September 2014.

[4] Benjamin W. Spencer, Wen Jiang, John E. Dolbow, and Christian Peco. Pellet cladding mechanical interaction modeling using the extended finite element method. In Proceedings of Top Fuel 2016, Boise, ID, September 2016.

[5] R.L. Williamson, K.A. Gamble, D.M. Perez, S.R. Novascone, G. Pastore, R.J. Gardner, J.D. Hales, W. Liu, and A. Mai. Validating the BISON fuel performance code to integral LWR experiments. Nuclear Engineering and Design, 301:232 - 244, 2016. 